University of Nebraska - Lincoln

DigitalCommons@University of Nebraska - Lincoln

Anthony F. Starace Publications

Research Papers in Physics and Astronomy

May 2005

\title{
Magnetic materials for finite-temperature quantum computing
}

\author{
Ralph Skomski \\ University of Nebraska-Lincoln, rskomski2@unl.edu \\ J. Zhou \\ University of Nebraska - Lincoln \\ A.Y. Istomin \\ University of Nebraska - Lincoln \\ Anthony F. Starace \\ University of Nebraska-Lincoln, astarace1@unl.edu \\ David J. Sellmyer \\ University of Nebraska-Lincoln, dsellmyer@unl.edu
}

Follow this and additional works at: https://digitalcommons.unl.edu/physicsstarace

Part of the Physics Commons

Skomski, Ralph; Zhou, J.; Istomin, A.Y.; Starace, Anthony F.; and Sellmyer, David J., "Magnetic materials for finite-temperature quantum computing" (2005). Anthony F. Starace Publications. 89.

https://digitalcommons.unl.edu/physicsstarace/89

This Article is brought to you for free and open access by the Research Papers in Physics and Astronomy at DigitalCommons@University of Nebraska - Lincoln. It has been accepted for inclusion in Anthony F. Starace Publications by an authorized administrator of DigitalCommons@University of Nebraska - Lincoln. 


\title{
Magnetic materials for finite-temperature quantum computing
}

\author{
R. Skomski, J. Zhou, A. Y. Istomin, A. F. Starace, and D. J. Sellmyer \\ Department of Physics and Astronomy and Center for Materials Research and Analysis, \\ University of Nebraska, Lincoln, Nebraska 68588
}

(Presented on 9 November 2004; published online 17 May 2005)

\begin{abstract}
The potential use of interacting magnetic nanodots for quantum computing (qubit) operations is investigated by model calculations. The quantum entanglement of the low-lying ferromagnetic states, as quantified by the concurrence, exhibits a resonant peak whose position and width depend on parameters such as dot anisotropy, interdot exchange, and external field gradient. The maximum operation temperature is proportional to the magnetocrystalline anisotropy of the dot material. A specific condition is that the dots are sufficiently small so that the interatomic exchange ensures a coherent magnetization state and quantum coherence at finite temperatures. From a material point of view, there is a quite rigid upper limit of about $100 \mathrm{~K}$, but to avoid decoherence it will be necessary to sacrifice a substantial fraction of this temperature, probably at least one order of magnitude. (C) 2005 American Institute of Physics. [DOI: 10.1063/1.1860832]
\end{abstract}

\section{INTRODUCTION}

The use of quantum bits (qubits) is a promising way to meet the ever-increasing needs of information technology, with various advantages over classical information processing in areas such as factorization and cryptography. ${ }^{1-3}$ In principle, any quantum-mechanical two-level system can be used as a qubit, and various physical realizations have been considered so far, such as electronic quantum wells and nuclear spins. Among the systems of current interest are spin chains and spin clusters, whose quantum states can be tuned, for example, by an external magnetic field. ${ }^{4-6}$

Most systems considered at present operate at very low temperatures, typically much smaller than $1 \mathrm{~K}$. The smallness of Bohr's magneton, $\mu_{B} / k_{B}=0.672 \mathrm{~K} / \mathrm{T}$, makes it difficult to exploit magnetic fields ${ }^{4}$ at temperatures significantly above $1 \mathrm{~K}$. Superconducting magnets are able to create fields much larger than $1 \mathrm{~T}(10 \mathrm{kOe})$, but they are very cumbersome and may not establish a practical alternative. Exchange anisotropy, as advocated in Refs. 4 and 5, is a conceivable alternative, but lowest-order exchange is isotropic, and the exchange anisotropies $J_{x x}-J_{z z}$ and $J_{y y}-J_{z z}$ are small and difficult-to-realize relativistic corrections to the isotropic exchange $\left(J_{x x}+J_{y y}+J_{z z}\right) / 3$.

Here we consider the use of coupled anisotropic magnetic nanodots for quantum information processing significantly above $4.2 \mathrm{~K}$. Emphasis is on the entanglement of the dots, which reflects the quantum-theoretical aspect of the problem, and the selection and development of optimized dot materials.

\section{NANODOT ENTANGLEMENT}

Figure 1 shows the considered two-dot model. There are two generally nonequivalent magnetic nanodots or clusters with total spins $S$ and $S^{\prime}$. The spins $S$ and $S^{\prime}$ are proportional to the dot size and can be written as $S=N S_{o}$ and $S^{\prime}=N^{\prime} S^{\prime}{ }_{o}$. In a simple interpretation, $S_{o}$ and $S^{\prime}{ }_{o}$ are the numbers of spins per atom and $N$ and $N^{\prime}$ are the numbers of atoms per dot. The main reason for considering nonequivalent dots is that real nanomagnets tend to have imperfections.

The single-dot spin Hamiltonians are of the type $\mathrm{H}_{o}$ $=\mathrm{H}_{a}-g \mu_{o} \mu_{B} H \hat{S}_{z}$, where $H=H_{z}$ is the external magnetic field and

$$
\mathrm{H}_{a}=-\frac{K N}{3 S^{2}}\left(3 \hat{S}_{z}^{2}-\hat{S}^{2}\right)
$$

is the magnetic anisotropy. In this equation, the uniaxial anisotropy constant $K$ reflects the chemical composition and crystal structure of the dots. ${ }^{7}$ The energy difference (level spacing) between the lowest two eigenvalues of Eq. (1), $K N(2 S-1) / S^{2}$, determines the maximum operation temperature. Since we consider two different dots, we must use two anisotropy constants $K$ and $K^{\prime}$. Note that the anisotropy leads to unequal level spacings, which simplify the addressing of well-defined quantum states, for example, in resonance experiments.

We assume that the two dots are coupled by a Heisenberg-type exchange $J$ realized, for example, by Ruderman-Kittel-Kasuya-Yosida (RKKY) interaction through a substrate or medium. ${ }^{8} J$ can be varied over many orders of magnitude, for example, by changing the dot distance, and roughly scales as some power of the dot size, $J$ $\sim N^{\alpha}{ }^{8,9}$ However, the origin of the coupling is of secondary interest in the present context; it may also be realized by a nanojunction or, with some modification of Eq. (2), by magnetostatic interactions.

A key aspect of quantum computing is the entanglement of the involved wave functions. ${ }^{4,10}$ Schrödinger's verschränkung or entanglement is a quantum effect without classical analog, a two-particle state being entangled if it cannot be expressed as a product state. In the Schmidt decomposition, the two-particle wave function can be written as 


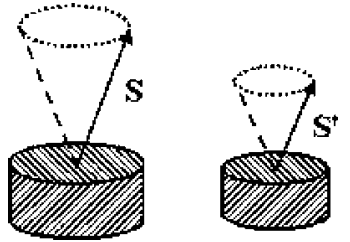

FIG. 1. Two interacting magnetic dots (schematic). The dots can be produced by a variety of deposition and fabrication methods, and the coupling may be realized by the net RKKY interaction through a nonmagnetic substrate or by a nanojunction.

$$
|\Psi\rangle=\alpha|00\rangle+\beta|01\rangle+\gamma|10\rangle+\delta|11\rangle .
$$

For example, the four maximally entangled Bell states are proportional to $|00\rangle \pm|11\rangle$ and $|01\rangle \pm|10\rangle$.

Since we are interested in low-temperature entanglement, we construct a Schmidt basis $|0\rangle$ and $|1\rangle$ from singledot states having $\left|S_{\mathrm{z}}\right|=S$ and $\left|S_{\mathrm{z}}\right|=S-1$, respectively. We consider only two states per dot: The ground-state $|0\rangle$ and the lowest-lying excited state $|1\rangle$. As shown elsewhere, ${ }^{11}$ higher excitations do not affect the entanglement of the low-lying states. Figure 2 illustrates the physical meaning of the four Schmidt states for the nanodot FM and AFM configurations.

For simplicity, we assume that the spin of the first dot is positive, so that the states $|0\rangle$ and $|1\rangle$ have the quantum numbers $S_{z}=S$ and $S_{z}=S-1$, respectively. For the second dot, the respective quantum numbers are $S_{z}^{\prime}=S^{\prime}$ and $S_{z}^{\prime}=S^{\prime}-1$ (FM) and $S^{\prime}{ }_{z}=-S^{\prime}$ and $S^{\prime}{ }_{z}=-S^{\prime}+1$. The matrix elements of $\mathrm{H}$ in terms of the Schmidt basis Eq. (2) are easily obtained by using the raising and lowering operators $\hat{S}^{ \pm}=\hat{S}_{x} \pm \mathrm{i} \hat{S}_{y}$ and $\hat{S}^{ \pm}=\hat{S}_{x}^{\prime} \pm \mathrm{i} \hat{S}^{\prime}{ }_{y}$.

A quantitative entanglement measure is the concurrence $C$. ${ }^{10}$ For nonentangled (separable) states, $C=0$, whereas the maximally entangled Bell states exhibit $C= \pm 1$. In the present case, the entanglement depends on whether the coupling is ferromagnetic $(\mathrm{FM}, J>0)$ or antiferromagnetic $(\mathrm{AFM}, J<0)$. The ferromagnetic ground state and the first two excited AFM states are separable, that is, $C=0$. Substantial entanglement is encountered in the AFM ground state and between low-lying ferromagnetic excitations ${ }^{11}$. Physically, the transverse components of the interdot exchange

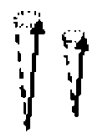

$100=$

(a)

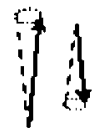

$100>$

(b)

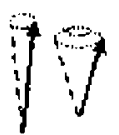

$01>$

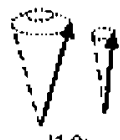

$110=$

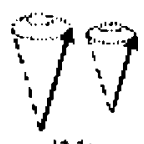

$\mid 11>$

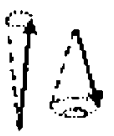

$01>$

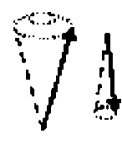

$110 \mathrm{p}$

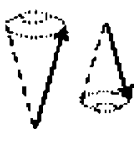

|1]>
FIG. 2. Low-lying states: (a) Ferromagnetic coupling and (b) antiferromagnetic coupling. As in Fig. 1, two coupled and generally inequivalent dots are considered, and the arrows denote to single-dot spins. The $\left|S_{z}\right|=S$ and $\left|S_{z}\right|$ $=S-1$ states are denoted by $|0\rangle$ and $|1\rangle$, respectively. (The $|0\rangle$ states have nonzero "cone angles" due to the quantum-mechanical uncertainty of the perpendicular spin components $S_{x}$ and $S_{y}$.)

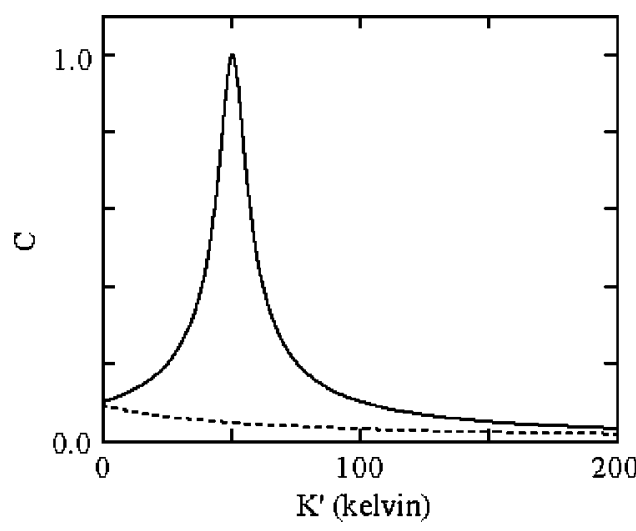

FIG. 3. Entanglement as a function of the anisotropy $K^{\prime}$ of the second dot. The solid and dashed lines denote FM and AFM couplings, respectively. The parameters are $S_{o}=S_{o}{ }^{\prime}=1, N=1000, N^{\prime}=1100, K=50 \mathrm{~K}$, and $J=0.005 \mathrm{~K}$. For simplicity, $H=0$ and $H^{\prime}=0$. The FM maximum is a resonance effect involving $|01\rangle$ and $|10\rangle$ states; there is no similar resonance in the AFM case.

give rise to mixing (and, hence, entanglement) of the states $|01\rangle$ and $|10\rangle(\mathrm{FM})$ and $|00\rangle$ and $|11\rangle(\mathrm{AFM})$.

Figure 3 shows the entanglement as a function of the anisotropy of the second dot. The entanglement of the lowlying FM excitations exhibits a resonant peak whose width depends on the interaction strength. Since a local magnetic field shifts the single-dot energies, a field gradient can be used to tune the entanglement. The dashed line in Fig. 3 shows that there is no peak in the antiferromagnetic case. Note that the present calculation is time-independent and does not aim at quantifying issues such as information input and output and decoherence.

\section{NANODOT MATERIALS}

Since the basic level splitting and the maximum operation temperature are proportional to $K$, it is necessary to exploit with high magnetocrystalline anisotropy. Dots with shape anisotropy and semihard dots, such as Co, cannot be used, because they correspond to temperatures of only about one kelvin. Figure 4 illustrates the role of the temperaturedependent anisotropy. On a phenomenological level, the maximum operating temperature is obtained graphically as the temperature at which the "architecture" line of the system (dashed) intersects the materials line (solid). The larger the slope of the architecture line, the lower the maximum application temperature. The slope reflects, in particular, quantum decoherence, reaching a minimum value of order $1 \mathrm{MJ} / \mathrm{m}^{3} \mathrm{~K}$ [line II in Fig. 4(a)] for systems where single-ion crystal-field excitations are the only consideration. For Co, the corresponding temperature is smaller than $1 \mathrm{~K}$, and similar values are encountered in other $3 d$ systems, such as ferritin.

Much higher design-dependent temperatures are obtained for $\mathrm{SmCo}_{5}$ [Fig. 4(a)]. However, typical hardmagnetic materials have temperature-dependent anisotropies that are maximized at or above room temperature. ${ }^{7,12}$ This includes not only rare-earth transition-metal alloys but also materials such $\mathrm{L} 1_{0}$ magnets, whose anisotropy originates from $4 d$ and $5 d$ atoms, such as Pd and $\mathrm{Pt}^{7}$ Little work has been done to optimize anisotropies at low temperatures, al- 

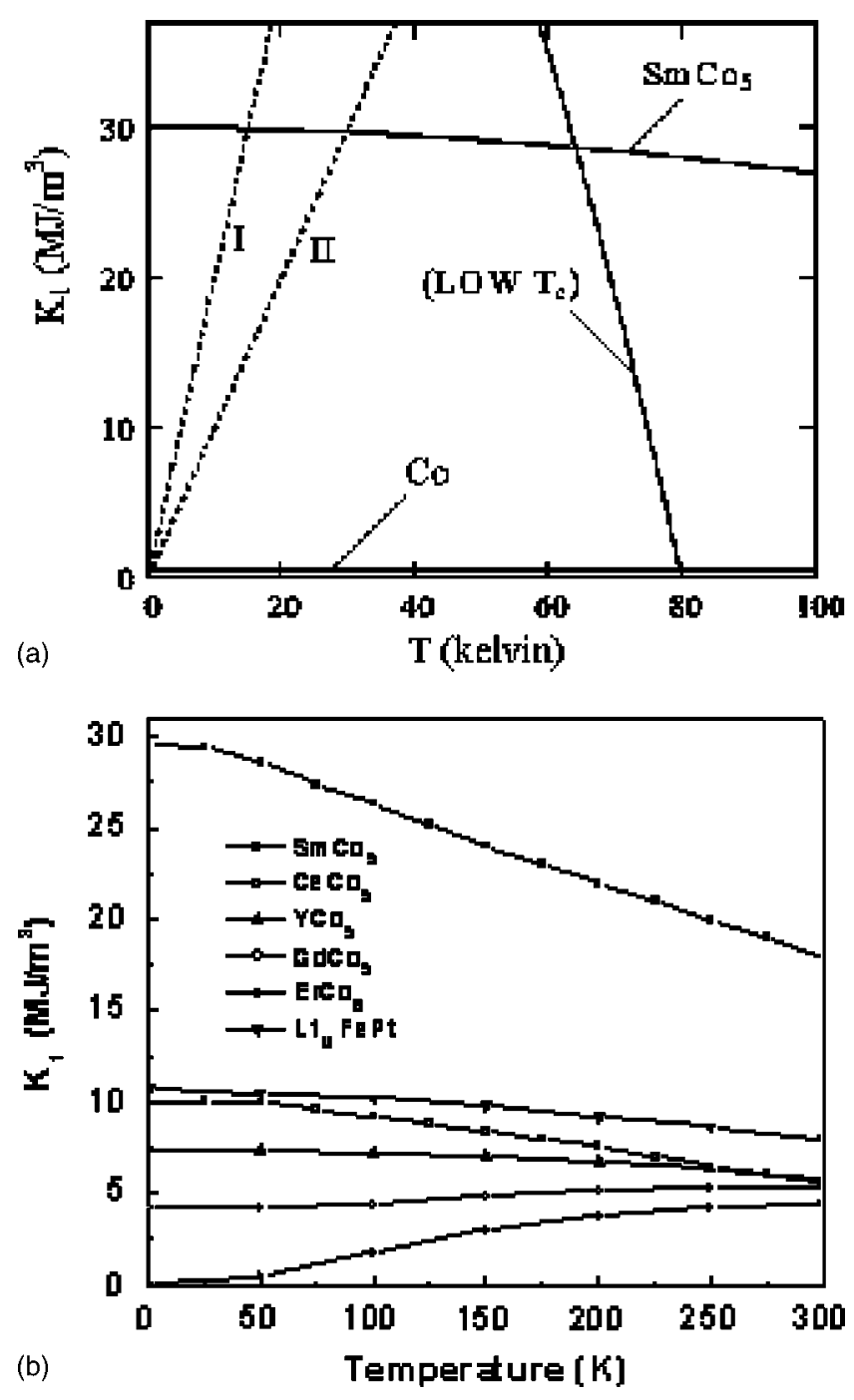

FIG. 4. Operating temperature and anisotropy: (a) Schematic theoretical figure and (b) experimental anisotropy data. (Ref. 15). In (a), the slopes of the dotted lines depend on the details of the coupling and geometry (decoherence): The well-optimized architecture II is more demanding than I but yields higher operating temperatures. The "low- $T_{c}$ " materials graph illustrates the effect of the Curie temperature $\left(K=0\right.$ at $\left.T_{c}\right)$.

though it is known that some compounds with $T_{c}<300 \mathrm{~K}$ have huge anisotropies of 100 to $1000 \mathrm{MJ} / \mathrm{m}^{3} .^{13,14}$ The low$T_{c}$ line in Fig. 4(a) is a typical example.

\section{DISCUSSIONS AND CONCLUSIONS}

As mentioned above, a field gradient can be used to tune the two-dot entanglement. However, issues such as initialization, readout, and specific gate implementations go beyond the scope of this paper. It is also necessary to avoid decoher- ence during the realization of the qubit operations. A key assumption of Secs. II and III is that the interatomic exchange yields well-defined good quantum numbers $S$ and $S^{\prime}$. This ensures coherence during qubit operations and determines, together with the time necessary to conduct an operation, the slope of dashed architecture line in Fig. 4(a).

An upper limit to the temperature above which spinwave-like excitations and magnetic domains destroy quantum coherence is given by the energies of the lowest-lying spin-wave states. They scale as $A a^{3} / L^{2}$, where $A$ is the exchange or spin-wave stiffness of the dot material, ${ }^{7} a$ is the interatomic distance, and $L$ is the dot size. For typical materials at temperatures significantly higher than $1 \mathrm{~K}$, this quantity does not exceed a few nanometers. In bigger dots, lowlying spin-wave excitations have energies comparable to $K$ and decoherence occurs very fast.

In conclusion, our concurrence calculations indicate that magnetocrystalline anisotropy can be used to realize qubit entanglement in realistic magnetic nanostructures. Low-lying ferromagnetic states exhibit a resonant quantum entanglement that can be tuned by varying the involved parameters, such as dot size, magnetocrystalline anisotropy, interdot coupling, and local magnetic field. An upper limit for the achievable operation temperature, significantly larger than $10 \mathrm{~K}$, is given by the anisotropy of the dots. A major condition for avoiding decoherence is to suppress spin waves by making the dots nanoscale rather than macroscopic.

\section{ACKNOWLEDGMENTS}

This work is supported by ARO, NSF MRSEC, the W. M. Keck Foundation, and CMRA.

${ }^{1}$ C. H. Bennet and D. P. DiVincenzo, Nature (London) 404, 247 (2000).

${ }^{2}$ A. Ekert and R. Josza, Rev. Mod. Phys. 68, 733 (1996).

${ }^{3}$ L. K. Grover, Phys. Rev. Lett. 79, 325 (1997).

${ }^{4}$ G. Lagmago Kamta and A. F. Starace, Phys. Rev. Lett. 88, 107901 (2002).

${ }^{5}$ F. Meier, J. Levy, and D. Loss, Phys. Rev. Lett. 90, 047901 (2003).

${ }^{6}$ J. Tejada, E. M. Chunovsky, E. del Barco, J. M. Hernandez, and T. P. Spiller, Nanotechnology 12, 181 (2001).

${ }^{7}$ R. Skomski and J. M. D. Coey, Permanent Magnetism (Institute of Physics, Bristol, 1999).

${ }^{8}$ R. Skomski, Europhys. Lett. 48, 455 (1999).

${ }^{9}$ R. Skomski, A. Kashyap, Y. Qiang, and D. J. Sellmyer, J. Appl. Phys. 93, 6477 (2003)

${ }^{10}$ S. Hill and W. K. Wootters, Phys. Rev. Lett. 78, 5022 (1997).

${ }^{11}$ R. Skomski, A. Y. Istomin, A. F. Starace, and D. J. Sellmyer, Phys. Rev. A 70, 062307 (2004).

${ }^{12}$ K. Kumar, J. Appl. Phys. 63, R13 (1988).

${ }^{13}$ M. S. S. Brooks and B. Johansson, in Handbook of Magnetic Materials, Vol. 7, edited by K. H. J. Buschow (Elsevier, Amsterdam), pp. 139-230.

${ }^{14}$ R. Skomski, J. Appl. Phys. 91, 8489 (2002).

${ }^{15}$ N. H. Hai, N. M. Dempsey, and D. Givord, IEEE Trans. Magn. 39, 2914 (2003); R. Ballou, J. Magn. Magn. Mater. 54-57, 465 (1986); A. S. Ermolenko, IEEE Trans. Magn. 12, 992 (1976) 